\title{
Novel dry powder inhaler formulation containing antibiotic using combined technology to improve aerodynamic properties
}

\author{
Rita Ambrus ${ }^{*}$, Edit Benke1, Árpád Farkas², Imre Balásházy², Piroska Szabó-Révész ${ }^{1}$ \\ ${ }^{1}$ Faculty of Pharmacy, Institute of Pharmaceutical Technology and Regulatory Affairs, \\ University of Szeged, Szeged, Hungary \\ ${ }^{2}$ Centre for Energy Research, Hungarian Academy of Sciences, Budapest, Hungary \\ *Corresponding author: \\ arita@pharm.u-szeged.hu
}

\begin{abstract}
Dry Powder Inhaler (DPI) could offer a propellant-free, easy-to-use powder form ensuring better stability than liquid dosage forms. Therefore the development of traditional carrier-based and carrier-free new generation systems is a determinative factor in the field of DPI formulation. The purpose of our research work was to combine these two systems, utilizing their beneficial properties to produce a novel pulmonary drug delivery system containing ciprofloxacin hydrochloride (CIP). Co-spray drying, surface smoothing and the preparation of an interactive physical mixture were applied as the technological procedures of sample preparation. The carrier-based and carrier-free formulations, as well as the developed novel product were compared to each other. Structural investigations were made by X-ray powder diffraction and micrometric properties (habit, bulk density) were determined. Particle interactions were also evaluated to investigate surface free energy, cohesive-adhesive forces, and spreading coefficient. In vitro aerodynamic properties (mass median aerodynamic diameter, fine particle fraction (FPF) and emitted dose of DPIs were measured using Andersen Cascade Impactor. A novel in silico Stochastic Lung Model was also used to quantify the amount of particles deposited at the target area. The novel-formulated composition presented amorphous spherical particles with an average size of about $2 \mu \mathrm{m}$. The in vitro aerodynamic investigations showed a variance in FPF as a function of formulation method (carrier-based: 24\%, carrier-free: $54 \%$ and applying the novel combination method: 63\%). The in silico deposition results were in line with the in vitro measurements and yielded increased lung doses for the sample prepared by the combined technology. This novel DPI formulation provides an opportunity for a more effective therapy with deeper deposition of CIP.
\end{abstract}

Keywords: combined technologies, DPI formulation; ciprofloxacin hydrochloride; interparticle interaction; aerosolization efficiency; in silico numerical model 


\section{Graphical abstract:}

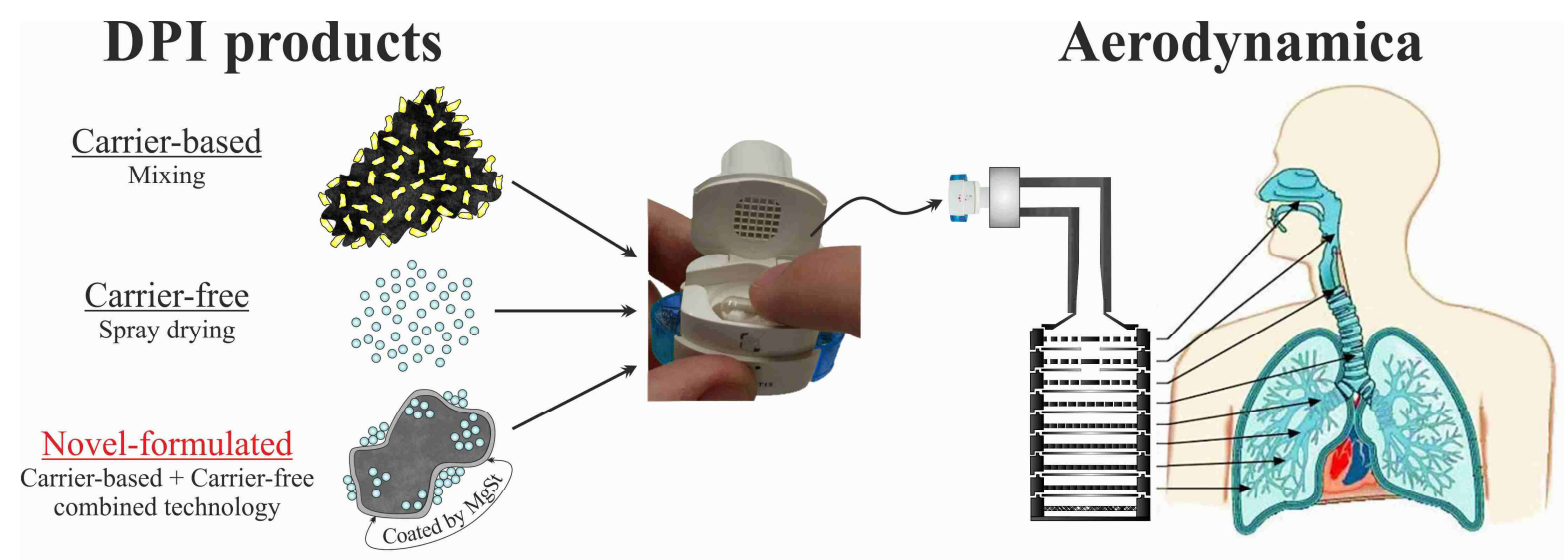




\section{Introduction}

Pulmonary drug administration enables to target drugs directly to the lung for local or systemic therapy. DPI compositions can be divided into two main categories, conventional carrier-based and carrier-free (new generation) systems (Benke et al., 2017). The marketed products belong to the traditional carrier-based formulations, applying a large carrier (e.g. lactose) and a micronized active pharmaceutical ingredient (Lee et al., 2018, Peng et al., 2016). Using a carrier is advantageous for active compounds with strong cohesive properties that have a positive effect on the flow properties of the product, the dosage of a small amount of drug can be refined or the taste of the carrier in the oral cavity during inhalation confirms the successful use of the product in the patient. However, most of these marketed products still have low pulmonary deposition values ( 20-30\% FPF, fine particle fractionation value), hence only a small percentage of the active ingredient can reach the proper lung segments because a large mass will be deposited in the upper respiratory tract. Only the modification of interparticle interaction (Tüske, 2005) (decrease of cohesive-adhesive forces by technological methods) could result in higher lung deposition. The role of the carrier and special additives applied in the designing phase is to prepare particles easy to handle during manufacturing and to provide an enhanced aerosolization property for inhalation (Velaga et al., 2018; Della Bella et al., 2017). Inhalac ${ }^{\circledR}$ series (different-sized lactose monohydrate, MEGGLE Group, Wasserburg, Germany) offer high quality drug transport to the lung (Pilcer et al., 2012). Lactose particles entering the lungs are rapidly absorbed, then metabolized and finally excreted in the urine. The processing of DPI formulation with a minimum concentration of magnesium stearate $(\mathrm{MgSt})$ is an effective means of improving the de-agglomeration and aerosolization of cohesive powders (Coccini et al., 2012). Making a thin film with this excipient could produce better aerosolization properties. It could be applied in a wide range between $0.001-10.0 \mathrm{w} / \mathrm{w} \%$. In the lower segments of the lung, only an insignificant amount of MgSt is released, which does not have negative effects and therefore many marketed products have a MgSt content (Palistra, 2008; Keller and MüllerWalz, 2003).

In the case of carrier-free DPI systems, the application of large carriers can be avoided with the use of special excipients and technologies (e.g. co-spray drying) (Chvatal et al., 2017; Yang et al., 2015). These systems yield around 50-60 \% FPF results due to the apparent high cohesive properties between the active ingredient's particles (Kadota et al., 2015). These formulations involve the usage of different amino acids (e.g. L-leucine) and polymers (e.g. polyvinyl alcohol or polyvinylpyrrolidone) (Pilcer and Amighi, 2010). The most important advantages of these novel formulations are their low density, improved aerodynamic properties and higher lung deposition (Healy et al., 2014). However, the main problem with the microparticles is that they stick to each other because of their high surface free energy. Overcoming this cohesive nature included the incorporation of large amounts of lipophilic adjuncts (such as the mixture of cholesterol and phospholipids e.g. sodium stearate) (Buttini et al., 2012). The preliminary analysis of the toxicity effect of sodium stearate on A549 lines showed that the adjunct, in the concentration (maximum $3 \mathrm{w} / \mathrm{w} \%$ ) used, had no effect on cell viability over a $24 \mathrm{~h}$ period compared to particles of pure tobramycin.

The use of antibiotics in the treatment of pulmonary fibrosis has long been known since Abbot Laboratories developed the inhaler (Aerohalor) containing penicillin powder in 1948. The most common treatment for bacterial respiratory infections is currently the oral administration of high doses of single or combined antibiotics, which may have serious side effects. With the development of antibiotic-containing DPI systems, the local treatment of these diseases is 
possible. Thus, high levels of the active substance can be achieved in the lung tissue, smaller doses are sufficient for oral therapy, leading to reduced systemic antibiotic exposure and lower toxicity. Ciprofloxacin hydrochloride (CIP) belongs to fluoroquinolones, it has a 6-fluoro-7piperazinyl group, which is responsible for its antibacterial effectiveness (Gram- and Gram+ microorganisms) (Karimi et al., 2016). CIP is a major advance in the treatment of bronchopulmonary infection in patients with cystic fibrosis and chronic obstructive lung disease. At present, tobramycin and aztreonam are the only inhaled antibiotics on the market. CIP is also a potential pharmacon for DPI formulation (it is in Phase III as a PulmoSphere ${ }^{\circledR}$ system) (Stass et al., 2008). Therefore it is important to study its newer DPI formulations.

The aim of our work is to develop a novel DPI system using combined technology to formulate CIP containing DPI samples. It was important that all of the formulated DPI samples (carrierbased, carrier-free and novel combined method) will be fulfilling the requirements of effective pulmonary drug delivery (micronized particles with low density for deep lung deposition). Our goal is to exceed the FPF values of the carrier-based and carrier-free DPIs and to find correlations between the interparticle interactions and the lung exposure results and to suggest a novel DPI formulation for pulmonary application of CIP.

\section{Materials and methods}

\subsection{Materials}

Micronized ciprofloxacin hydrochloride ( $\mu$ CIP), a fluoroquinolone-type antibiotic, (D50: 5.09 $\mu \mathrm{m})$ was kindly supplied by Teva Pharmaceutical Works Ltd. (Debrecen, Hungary). Lactose monohydrate, Inhalac ${ }^{\circledR} 70$ (IH 70) (D50: $215.00 \mu \mathrm{m}$ ) (MEGGLE Group, Wasserburg, Germany) was used as a carrier base. Magnesium stearate (MgSt) was applied as a surface modifier (Sigma-Aldrich, Budapest, Hungary) (D50: $6.92 \mu \mathrm{m}$ ). Sodium stearate (NaSt) (Alfa Aesar, Heysham, United Kingdom) was used for habit modification.

\subsection{Methods}

\subsubsection{Preparation of dry powder formulation}

Figure 1. presents the procedures which were used for carrier-based, carrier-free and their combined method for DPI preparation.

First, we applied a well-known formulation method. According to the preliminary experiments, a large size Inhalac ${ }^{\circledR}$ type, IH 70 was chosen for the carrier-based formulation. These samples were prepared by mixing (Turbula System Schatz; Willy A. Bachofen AG Maschinenfabrik, Basel, Switzerland) using 1:10 drug : IH70 ratio (Benke et al., 2017) at $60 \mathrm{rpm}$ for 30 minutes.

The carrier-free formulation applied a co-spray-drying method with NaSt and the use of 30\% of EtOH as the solvent phase to modify the habit of CIP. CIP solution to be spray-dried was prepared by mixing a $70 \mathrm{~g}$ of CIP containing aqueous solution $(1.5 \mathrm{w} / \mathrm{v} \%)$ with a $30 \mathrm{~g}$ of NaSt $(0.0175 \% \mathrm{w} / \mathrm{v})$ containing alcoholic solution applying $30^{\circ} \mathrm{C}$. Finally, the prepared solid samples had $99.5 \%$ of CIP and $0.5 \%$ of NaSt content. The spray drying procedure was carried out using a Büchi B-191 apparatus (Mini Spray Dryer, Büchi, Switzerland). The drying parameters were adjusted as follows, based on our previous experiments, see Table 1. (Pomázi et al., 2014).

A combination of these two methods completed with surface smoothing of $\mathrm{IH} 70$ by $\mathrm{MgSt}$ (modification of the carrier's surface and blending with a co-spray dried CIP system) resulted in the novel DPI formulation. The surface modification was made by $2 \%$ of MgSt with mixing 
for $4 \mathrm{~h}$ in a Turbula mixer. The carrier-free formulation containing $99.5 \%$ of CIP and $0.5 \%$ NaSt was prepared by spray drying then mixing for 30 minutes with IH70_MgSt as modified carrier in a 1:10 ratio as a combination method (see Table 1 ).

1)

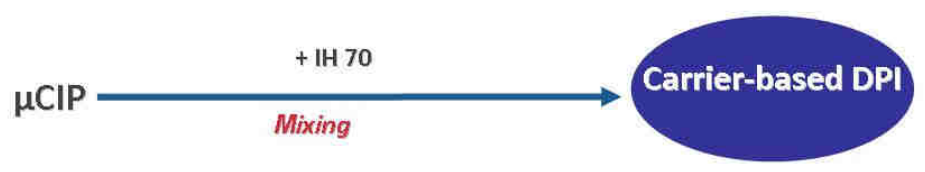

2)

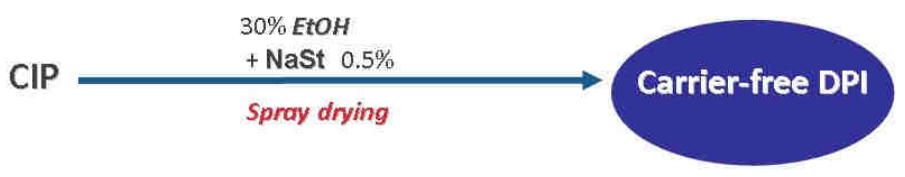

3)

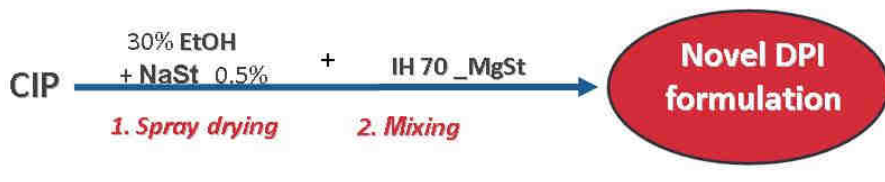

\section{Figure 1. Preparation protocol for different types of DPI formulations}

Table 1. focuses on and presents the selected compositions and Table 2. their process parameters as regards carrier-based, carrier-free and novel combined formulations.

\section{Table 1. Composition of the products}

\begin{tabular}{|c|c|c|c|}
\hline & $\begin{array}{c}\text { 1, Carrier-based DPI } \\
(\mu \mathrm{CIP}+\mathrm{IH70})\end{array}$ & $\begin{array}{l}\text { 2, Carrier-free DPI } \\
\text { (CIP_0.5NaSt_spd) }\end{array}$ & $\begin{array}{l}\text { 3, Novel DPI formulation } \\
\text { (CIP_0.5NaSt_spd+IH70_MgSt) }\end{array}$ \\
\hline \multirow[t]{6}{*}{ Compositions } & CIP-1.05 g & CIP-1.05 g & CIP-1.05 g \\
\hline & & NaSt-0.00525 g & NaSt-0.00525 g \\
\hline & & $30 \%$ EtOH- ad $100 \mathrm{~g}$ & $30 \%$ EtOH- ad $100 \mathrm{~g}$ \\
\hline & IH70-10.50 g & & + \\
\hline & & & IH70-10.32 g \\
\hline & & & MgSt-0.232 g \\
\hline
\end{tabular}


Table 2. Process parameters of the products

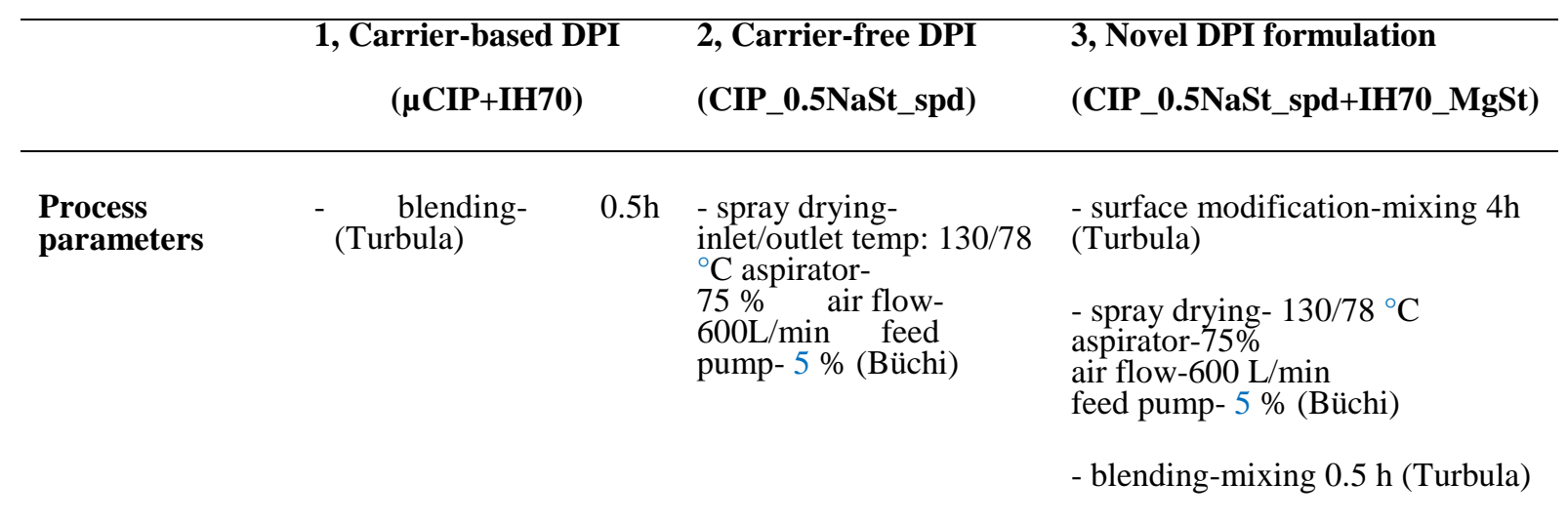

\subsubsection{X-ray powder diffraction (XRPD)}

The solid state form of the formulations was characterized by a BRUKER D8 Advance X-ray powder diffractometer (Bruker AXS GmbH, Karlsruhe, Germany) with $\mathrm{Cu} K \lambda_{\mathrm{I}}$ radiation $(\lambda=$ $1.5406 \AA$ ) and a VÅNTEC-1 detector. The powder samples were scanned at $40 \mathrm{kV}$ and $40 \mathrm{~mA}$, with an angular range of $3^{\circ}$ to $40^{\circ} 2 \theta$, at a step time of $0.1 \mathrm{~s}$ and a step size of $0.01^{\circ}$.

\subsubsection{Micrometric properties}

\subsubsection{Scanning electron microscopy (SEM)}

Scanning electron microscopy (SEM) (Hitachi S4700, Hitachi Scientific Ltd., Tokyo, Japan) was applied for the control of the morphology at $10 \mathrm{kV}$. The samples were coated by goldpalladium (90 seconds) with a sputter coater (Bio-Rad SC 502, VG Microtech, Uckfield, UK) using an electric potential of $2.0 \mathrm{kV}$ at $10 \mathrm{~mA}$ for $5 \mathrm{~min}$. The air pressure was $1.3-13.0 \mathrm{MPa}$.

\subsubsection{Particle size and distribution}

The particle size of the samples was determined by laser diffraction (Malvern Mastersizer Scirocco 2000; Malvern Instruments Ltd., Worcestershire, UK). The dispersion medium was air for the characterized microparticles. The particle size distribution was characterized by the $\mathrm{D}(0.1), \mathrm{D}(0.5)$, and $\mathrm{D}(0.9)$ values and the specific surface area.

\subsubsection{Determination of bulk density}

The bulk density of the samples was determined using a $5 \mathrm{ml}$ tared graduated cylinder, filled with powder. After direct mass and volume measurement, the following relationship was applied: mass/volume.

\subsubsection{Interparticle interactions}

After the determination of the contact angle, surface free energy and polarity could be calculated. Around $0.15 \mathrm{~g}$ of the sample on 1 ton hydraulic press (Perkin Elmer hydraulic press, Waltham, USA) was pressed to pastille. Then the surface of the pastilles was dripped with polar and non-polar solvents. The contact angle was detected for $30 \mathrm{sec}$ with Dataphysics OCA 20 apparatus (Dataphysics Inc. GmbH, Germany), then the Wu-correlation was used. 
The surface free energy of the material $\left(\gamma_{s}\right)$ is defined as the free energy change that occurs when the surface is increased one unit. It consists of two parts: a disperse part $\left(\gamma_{s}^{d}\right)$ and a polar part $\left(\gamma_{s}^{p}\right)$, so the following formula could be used for calculation $\left(\gamma_{s}=\gamma_{s}^{d}+\gamma_{s}^{p}\right)$. The solvents were distilled water $\left(\gamma^{\mathrm{p}}=50.2 \mathrm{mN} / \mathrm{m}, \gamma^{\mathrm{d}}=22.6 \mathrm{mN} / \mathrm{m}\right)$ and diiodomethane $\left(\gamma^{\mathrm{P}}=1.8 \mathrm{mN} / \mathrm{m}, \gamma^{\mathrm{d}}=49\right.$ $\mathrm{mN} / \mathrm{m})$.

If the surface free energy $\left(\gamma_{s}\right)$ and the polar component $\left(\gamma_{s}^{p}\right)$ are known, the polarity of the samples can be determined according to the following equation $\left(\gamma_{s}^{p} / \gamma_{s}\right)^{*} 100$.

The work of cohesion is determined from the surface free energy:

$$
\mathrm{W}_{\mathrm{c}}=2 * \gamma_{\mathrm{s}}
$$

The work of adhesion $\left(\mathrm{W}_{\mathrm{a}}\right)$ is equal numerically to the energy that arises when two surfaces come into contact [92-94]:

$$
\mathrm{W}_{\mathrm{a}}=4\left[\frac{\gamma_{1}^{\mathrm{d}} \gamma_{2}^{\mathrm{d}}}{\gamma_{1}^{\mathrm{d}}+\gamma_{2}^{\mathrm{d}}}+\frac{\gamma_{1}^{\mathrm{p}} \gamma_{2}^{\mathrm{p}}}{\gamma_{1}^{\mathrm{p}}+\gamma_{2}^{\mathrm{p}}}\right]
$$

The force of adhesion was calculated by Derjaguin hypothesis $\left(\mathrm{F}_{\mathrm{adh}}\right)[18]$ :

$$
F_{\text {adh }}=2 \pi\left(\frac{R_{A} R_{B}}{R_{A}+R_{B}}\right) W_{a d h}
$$

where $R_{A}$ and $R_{B}$ are the radius of particle $A$ and $B$, and the adhesive interaction was measured between them. $R$ was determined during particle size analyses ( $D[0.5] / 2)$.

The spreading coefficient $\left(S_{12}\right)$ shows the probability of one material (1) on the surface of the other material (2). It is used to characterize the distribution in two-component systems. This is a dimensionless number. The spread is more favourable and energy-efficient if the coefficient is a positive value and the number is larger. In our case, the spreading of the active ingredient can be characterized on the surface of the large substrate. The coefficient can be calculated with the following equations:

$$
\begin{gathered}
\mathrm{S}_{12}=4\left[\frac{\gamma_{1}^{\mathrm{d}} \gamma_{2}^{\mathrm{d}}}{\gamma_{1}^{\mathrm{d}}+\gamma_{2}^{\mathrm{d}}}+\frac{\gamma_{1}^{\mathrm{p}} \gamma_{2}^{\mathrm{p}}}{\gamma_{1}^{\mathrm{p}}+\gamma_{2}^{\mathrm{p}}}-\frac{\gamma_{1}}{2}\right] \\
\mathrm{S}_{21}=4\left[\frac{\gamma_{1}^{\mathrm{d}} \gamma_{2}^{\mathrm{d}}}{\gamma_{1}^{\mathrm{d}}+\gamma_{2}^{\mathrm{d}}}+\frac{\gamma_{1}^{\mathrm{p}} \gamma_{2}^{\mathrm{p}}}{\gamma_{1}^{\mathrm{p}}+\gamma_{2}^{\mathrm{p}}}-\frac{\gamma_{2}}{2}\right]
\end{gathered}
$$

where $\gamma^{\mathrm{d}}$ is the disperse part of the surface free energy and $\gamma^{\mathrm{p}}$ is the polar part of the surface free energy and $\gamma$ is the total surface free energy of the components whose is spread on the other component (Tüske, 2005).

\subsubsection{In vitro-In silico aerodynamic deposition}




\subsubsection{In vitro deposition by Andersen Cascade Impactor}

In the field of pharmaceutical industry, the Andersen Cascade Impactor is a vertical equipment most generally used for the in vitro testing of the aerodynamic properties of inhaled DPIs. It complies with the specifications laid down in Ph. Eur. 2.9.18 (European Pharmacopoeia, 2015). The standard Andersen Cascade Impactor is designed for use with a flow rate at $28.3 \mathrm{~L} / \mathrm{min}$ by a vacuum pump (High-capacity Pump Model HCP5, Critical Flow Controller Model TPK, Copley Scientific Ltd., Nottingham, UK). The actual flow rate through the impactor was detected by a mass flow meter (Flow Meter Model DFM 2000, Copley Scientific Ltd., Nottingham, UK) for each run. Its 8 stages are available for the particle size collection (Wong et al., 2010) where particles deposited at stages 2-5 are in the size range of $4.7-1.1 \mu \mathrm{m}$ suitable for effective inhalation. The stages were coated with a $1 \% \mathrm{~m} / \mathrm{m}$ mixture of $\operatorname{Span}^{\circledR} 80$ and cyclohexane to simulate pulmonary adhesive circumstances, in order to allow for the attachment of floating particles and to imitate the surface humidity of the airways. Three parallel measurements were done, each time using hard gelatine capsules (transparent, size 3, Capsugel, Germany) to be 'inhaled' at the appropriate flow rate. Each capsule contained different amounts of powder sample equalling $10 \mathrm{mg}$ of CIP, which is $10 \%$ of the oral dose as recommended. A Breezhaler ${ }^{\circledR}$ (Novartis) as DPI device was used for the inhalations. The inhaler, the mouthpiece, the induction port and each of the eight stages were washed out with distilled water to collect the deposited CIP. The drug concentration was quantified by an Ultraviolet-visible spectrophotometer (ATI-UNICAM UV/VIS Spectrophotometer, Cambridge, UK) at $276 \mathrm{~nm}$. Fine particle fraction (FPF), mass median aerodynamic diameter (MMAD) and emitted dose (ED) were determined to characterize the deposition profile of the prepared DPI. The FPF (particles $<5 \mu \mathrm{m}$ ) is representative of those particles that have a high probability of penetrating into the bronchial region and (particles $<3 \mu \mathrm{m}$ ) deposit in the deep lung. The MMAD corresponds to the diameter of the particles deposited in the impactor for which $50 \% \mathrm{w} / \mathrm{w}$ of particles have a lower diameter and $50 \% \mathrm{w} / \mathrm{w}$ have a higher diameter (Parlati 2008). ED is the dose (mg) which leaves the device and it is calculated by weight differences of the inhaler device before and after drug delivery.

\subsubsection{In silico stochastic model}

The newest version of the Stochastic Lung Model (SLM) (Koblinger and Hofmann, 1990) has been applied to quantify the amount of inhaled drug depositing in the upper airways and in the lungs. Regional deposited dose fractions were defined as the mass of drug deposited in a given region (extrathoracic or lungs) expressed as a percent of the mass of drug metered in the capsule. The deposition was modelled by tracking the individual drug particles within the airways until they deposit or are exhaled. Inertial impaction, gravitational settling and thermal diffusion deposition mechanisms were considered. Deposition computations in the upper airways were based on empirical formulas (Cheng 2003). The tracheobronchial part of the haefelairways was modelled based on the database of Raabe et his co-workers containing morphometrical parameters such as tube lengths and diameters and branching and gravity angles. The geometry of the acinar airways was based on the data (Haefeli-Bleuer and Weibel, 1988). The numerical deposition model was validated for the case of medical aerosols in our earlier works (e.g. Farkas et al. 2016; Farkas et al. 2017). The inputs of the model were the size distributions of the drugs measured in this work by cascade impactor techniques and the inhalation profile of a COPD patient when inhaling through Breezhaler ${ }^{\circledR}$ derived from the work (Colthorpe et al. 2013). This profile (patient 7 in the work (Colthorpe et al. 2013)) characterized by an inhaled volume of $1700 \mathrm{ml}$ and inhalation time of $3.2 \mathrm{~s}$ was chosen because the corresponding flow rate fits well with the flow rate through the impactor applied by us for the determination of particle size distributions (30 L/min). It is worth noting that since the drugs are emitted by a DPI, both the 
emitted dose and particle size distribution would be different at another flow rate.

\section{Results and discussion}

\subsection{Structural investigations}

By X-ray (XRPD) we obtained useful information about the excipients and particle engineered materials for DPI formulation. The knowledge of the solid state form (crystalline/amorphous character) of CIP in the product can be determinative in terms of aerodynamic behavior, efficiency, compatibility with excipients and stability of drug formulation during storage.

To evaluate the patterns of the excipients (Figure 2., upper part) we can conclude that $\mathrm{MgSt}$ (3.8 and 5.5 2Theta degree) and NaSt (4.0 and 6.0 2Theta degree) are crystalline materials. IH 70 is crystalline with $12.8,16.8$ and 20.0 2Theta degree, which solid state form did not change after its surface modification using $4 \mathrm{~h}$ mixing. However, the low concentration of $\mathrm{MgSt}$ did not appear on the XRPD pattern of IH 70_MgSt, which means that the produced thin film of MgSt covered the surface of IH 70. According to the literature (Byrn et al. 2017), the lactose particles can be broken by mechanical forces (e.g. mixing for a long time) and this can result in a decrease in crystallinity. We concluded that the crystallinity of IH 70 was the same as before the surface modification.

Based on the results of XRPD (Figure 2., lower part), the crystalline character of untreated $\mu \mathrm{CIP}$ can be seen. The fingerprint shows a number of typical peak intensities that exhibit high crystallinity, pattern with peak intensity at 8.2, 9.5, 19.22, 26.39 and 29.16 2Theta degree. During the preparation of the carrier-based system $(\mu \mathrm{CIP}+\mathrm{IH} 70)$, the crystalline character of CIP and IH 70 was retained. However, in the case of the carrier-free system (CIP_0.5NaSt_spd), the amorphous nature of CIP could be detected by the presence of NaSt and using the spray drying method. We observed the same amorphous character if CIP and the crystalline state of IH70_MgSt produced the novel DPI by the combined technology (CIP_0.5NaSt_spd+IH70_MgSt). 

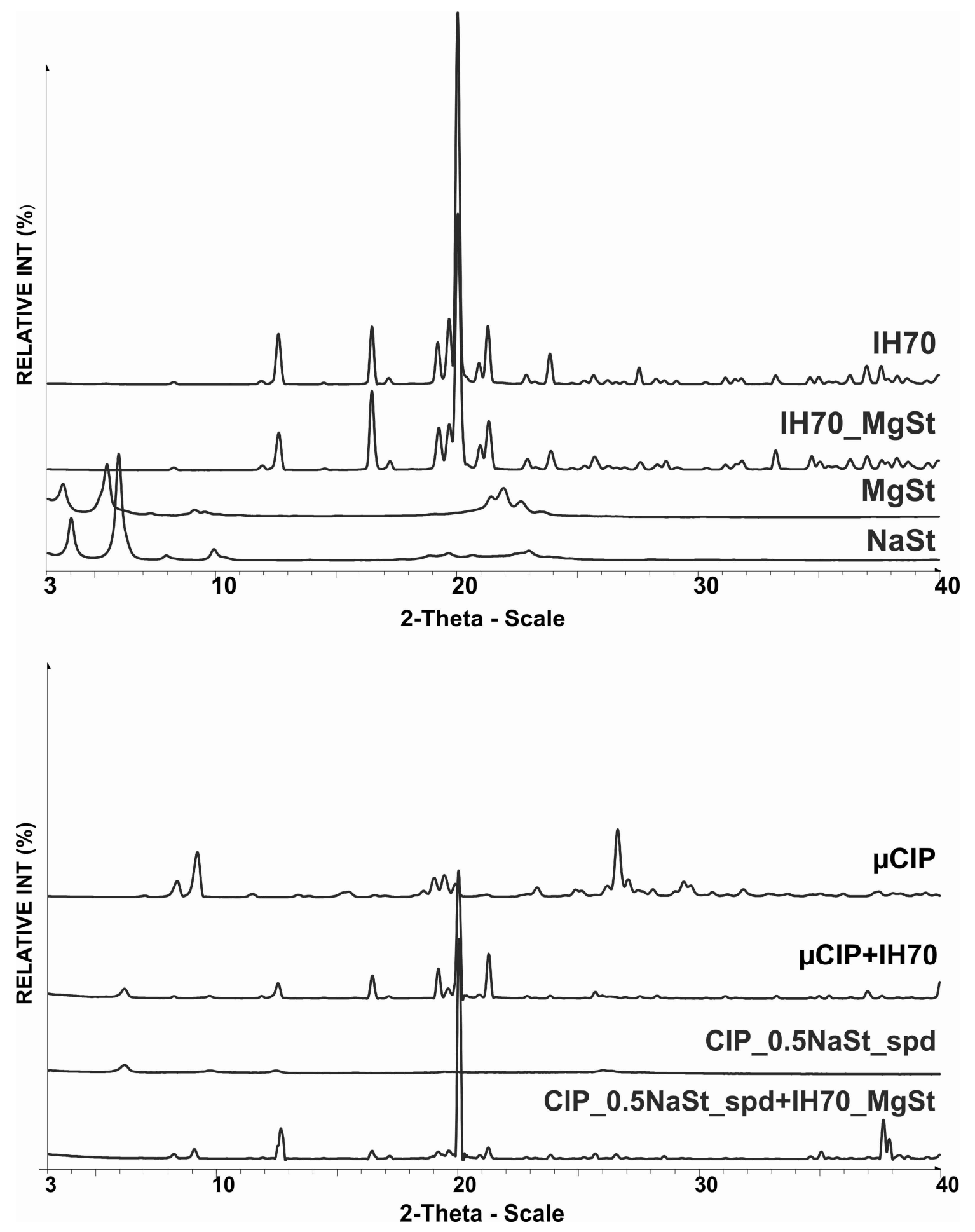

Figure 2. XRPD pattern of excipients and applied CIP for DPI formulations

\subsection{Evaluation of the micrometric properties}

The individual surface properties, the morphology of the raw materials, as well as the distribution of the CIP on the surface of the carrier were investigated by SEM pictures (Fig. 3.). The analyses of IH 70 revealed large, smooth, prismatic, nearly rhomboid particles in the image. We concluded that $\mu$ CIP showed small crystals with an irregular shape. The surface of IH70 had more active places offering insertions for the microsized drug particles, which presented uniform distribution on the surface $(\mu \mathrm{CIP}+\mathrm{IH} 70)$. Spray dried samples contained monodisperse 
particles with spherical morphology with surface roughness. A small amount of NaSt could help to produce individual particles (CIP_0.5NaSt_spd). In the case of the combined technology, the presence of MgSt covered the active places on the surface of IH70, presenting a smooth area. Therefore the spherical particles concentrated in the cavities had heterogeneous distribution.
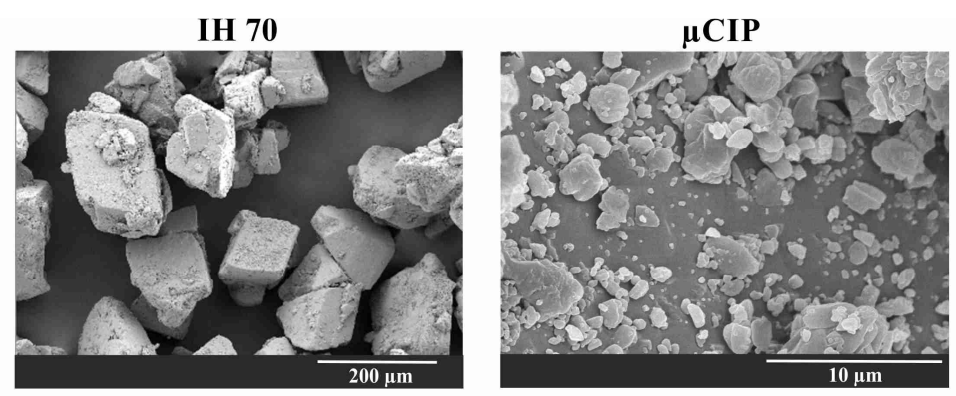

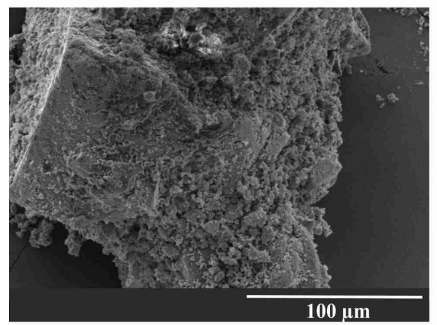

$\mu \mathrm{CIP}+\mathrm{IH} 70$

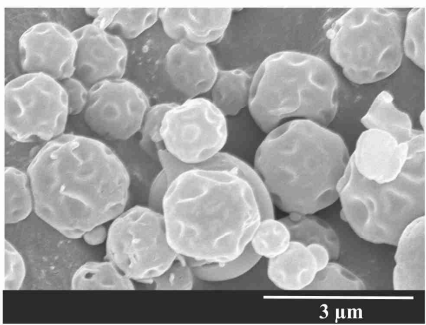

CIP_0.5NaSt_spd

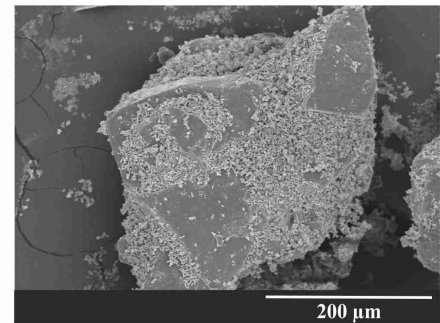

CIP_0.5NaSt_spd+IH70_MgSt

Figure 3. The morphology of raw IH70, $\mu$ CIP and the carrier-based, carrier-free, novel DPI formulations

Based on the results of particle size analyses (shown in Table 3.) it could be seen that IH 70 consisted of large particles $(215 \mu \mathrm{m})$ and the presence of $\mathrm{MgSt}$ with a $4 \mathrm{~h}$ smoothing procedure broke down the particles slightly and also increased the density of the carrier. $\mu$ CIP particles are in a range between $2-15 \mu \mathrm{m}$, they do not fully meet the size range requirement $(1-5 \mu \mathrm{m})$ for inhalation therapy, but the mean size is around $5 \mu \mathrm{m}$. Spray drying modified the size to be favourable $(1-5 \mu \mathrm{m})$. The determination of bulk density was determinative for $\mu$ CIP and CIP_0.5NaSt_spd because only the small drug particles will be deposited in the lung. We conclude that one-fourth density value can be achieved by particle engineering, which can offer improved aerodynamic properties for co-spray dried samples.

Table 3. Particle size distribution and bulk density of the samples

\begin{tabular}{|c|c|c|c|c|}
\hline & $\begin{array}{l}\mathrm{D} 0.1 \\
{[\mu \mathrm{m}]}\end{array}$ & $\begin{array}{l}\mathrm{D} 0.5 \\
{[\mu \mathrm{m}]}\end{array}$ & $\begin{array}{l}\text { D0.9 } \\
{[\mu \mathrm{m}]}\end{array}$ & $\begin{array}{c}\text { Bulk density } \\
\text { [g/cm3] }\end{array}$ \\
\hline IH70 & 135.02 & 215.00 & 305.34 & 0.600 \\
\hline IH70_MgSt & 71.48 & 174.32 & 286.01 & 0.7474 \\
\hline$\mu \mathrm{CIP}$ & 2.09 & 5.05 & 14.55 & 0.4192 \\
\hline$\mu \mathrm{CIP}+\mathrm{IH70}$ & 25.55 & 170.36 & 319.90 & - \\
\hline CIP_0.5NaSt_spd & 1.15 & 2.24 & 4.55 & 0.1459 \\
\hline
\end{tabular}


- not measurable

\subsection{In vitro aerodynamic measurement}

The aerodynamic characteristics were measured at $28.3 \mathrm{~L} / \mathrm{min}$ flow rates. Table 4 . shows the median mass aerodynamic diameter (MMAD) values, fine particle fraction (FPF) as well as the emitted dose (ED). The carrier-based formulation is correlated with the marketed products. With 7-9 $\mu$ m MMAD value only 23-24 \% FPF was produced. It means the drug will be deposited in the upper bronchial part of the lung. The carrier-free system, CIP_0.5NaSt_spd could be characterized by improved aerodynamic properties (FPF: 48-54\%). It means that the modified habit is more favourable than the irregular microsized CIP on the carrier. The novel combination method presented the lowest MMAD (around $3 \mu \mathrm{m}$ ). Because of the $63.75 \% \mathrm{FPF}$ $(<5 \mu \mathrm{m})$ and $39.22 \% \mathrm{FPF}(<3 \mu \mathrm{m})$ this novel formulation method offers an effective CIP dose in the deeper alveolar part of the lung, where the liquidation of a bacterial biofilm is very important. The ED by all of the samples were also high (around 90\%), indicating a favourably weak adhesive character between the powder and the capsule.

Table 4. Parameters determining in vitro lung deposition

\begin{tabular}{lcccc}
\hline Samples & $\begin{array}{c}\text { MMAD } \\
(\boldsymbol{\mu m})\end{array}$ & $\begin{array}{c}\text { FPF (\%) } \\
<\mathbf{5} \boldsymbol{\mu m}\end{array}$ & $\begin{array}{l}\text { FPF (\%) } \\
<\mathbf{3} \boldsymbol{\mu m}\end{array}$ & $\begin{array}{c}\text { ED } \\
(\boldsymbol{\%})\end{array}$ \\
\hline $\boldsymbol{\mu C I P + I H 7 0}$ & $7.9 \pm 0.10$ & $23.30 \pm 0.23$ & $11.88 \pm 0.20$ & $96.92 \pm 0.11$ \\
\hline CIP_0.5NaSt_spd & $4.14 \pm 0.18$ & $54.27 \pm 2.75$ & $27.14 \pm 2.38$ & $76.99 \pm 3.32$ \\
\hline CIP_0.5NaSt_spd+IH70_MgSt & $3.47 \pm 0.02$ & $63.75 \pm 1.21$ & $39.22 \pm 0.74$ & $90.45 \pm 1.80$
\end{tabular}

\subsection{In silico assessment for prediction particle deposition in the lung}

The breathing parameter values needed for the in silico simulations were: $1.7 \mathrm{~L}, 3.2 \mathrm{~s}$ and 5, 10 $\mathrm{s}$ for the inhaled volume, inhalation time and breath-hold time, respectively. These values correspond to a mean inhalation flow rate of $31.9 \mathrm{~L} / \mathrm{min}$. The in silico analyses were applied for the best DPI products including carrier-based, carrier-free and combined formulations. The results of the deposition simulations are shown in Figure 5. It is worth noting that all dose values in Figure 4. are expressed as a percent of drug dose metered in the capsule. We conclude that the deposition results of in vitro and in silico investigations using a $5 \mathrm{~s}$ breath-hold time exhibit the same tendency. The in silico deposition pattern for the carrier-based formulation contains a high extrathoracic dose fraction because of the significant number of large particles, which deposit by impaction mostly in the throat region. A high fraction of the particles are also exhaled, while lung deposition is the lowest for this formulation. For the carrier-free formulation, the deposition profile was much more convenient with a dose fraction of around $48 \%$ in the lung and a dose fraction of $12 \%$ as the exhaled amount. However, when using the combined procedure, the dose fraction in the lung was as high as $55 \%$. To assess the effect of the length of breath-holding after the inhalation of the drug, we calculated the regional deposited dose fractions also for a 10s breath-hold time. Figure 4. demonstrates that longer 
breath-hold results in higher lung doses and lower exhaled doses for all formulations. It means that with the special inhalation technique drug deposition can be further improved and the exhaled amount of drug kept at a minimum level, especially in the case of carrier-free samples and samples prepared by the combined method.

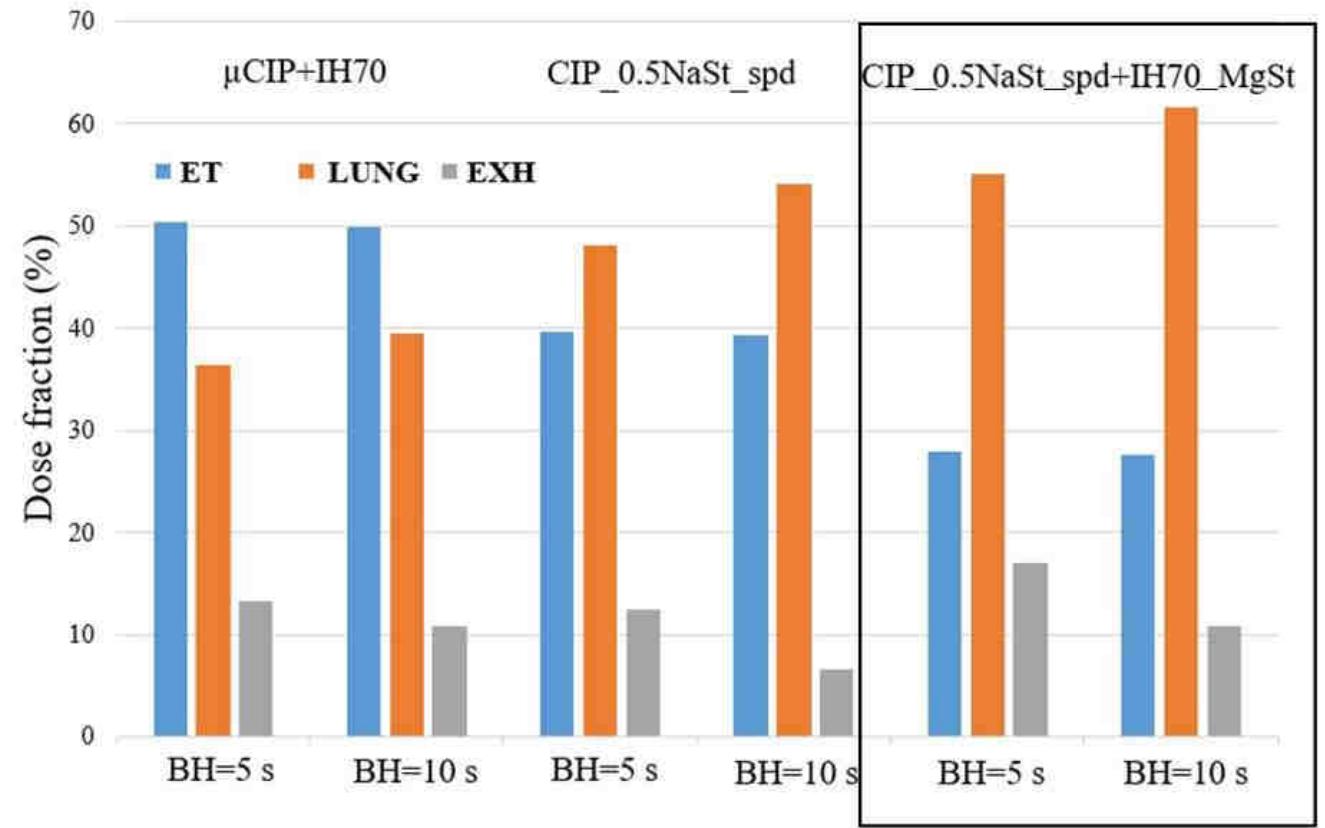

Figure 4. Comparison of the three formulation methods in silico by different breathhold (BH) times (ET: extrathoracic airways, LUNG: bronchial and acinar parts, EXH: exhalation fraction)

\subsection{The effect of interparticle interactions on aerodynamic properties}

The effectiveness of the novel DPI formulation developed with the combined technology can be related to the interparticle interactions between components, which modify the balance of cohesive and adhesive forces.

Without surface modification (Figure 5A) a microporous (pore diameter 1-10 $\mu \mathrm{m}$ ), by surface treatment (Figure 5B) a macroporous (pore diameter above $10 \mu \mathrm{m}$ ) surface of the applied IH70 could be found (Shalas et al., 2015). This also explains the phenomenon of the spread of different active substances. Therefore nearly homogeneous distribution was reached after blending $\mu$ CIP particles with IH70 (Figure 5A). Electron microscopic images (Figure 5B) were also made to study how MgSt affects the surface of the carrier and thus its role in the DPI formulation. It was found that MgSt modified (by thin film forming) the active sites of the carrier and thus altered the surface of the drug carrier, thereby presumably affecting the 
interparticle interactions. The spray-dried particles centered to different parts of the smoothed carrier because of the presence of $\mathrm{MgSt}$.

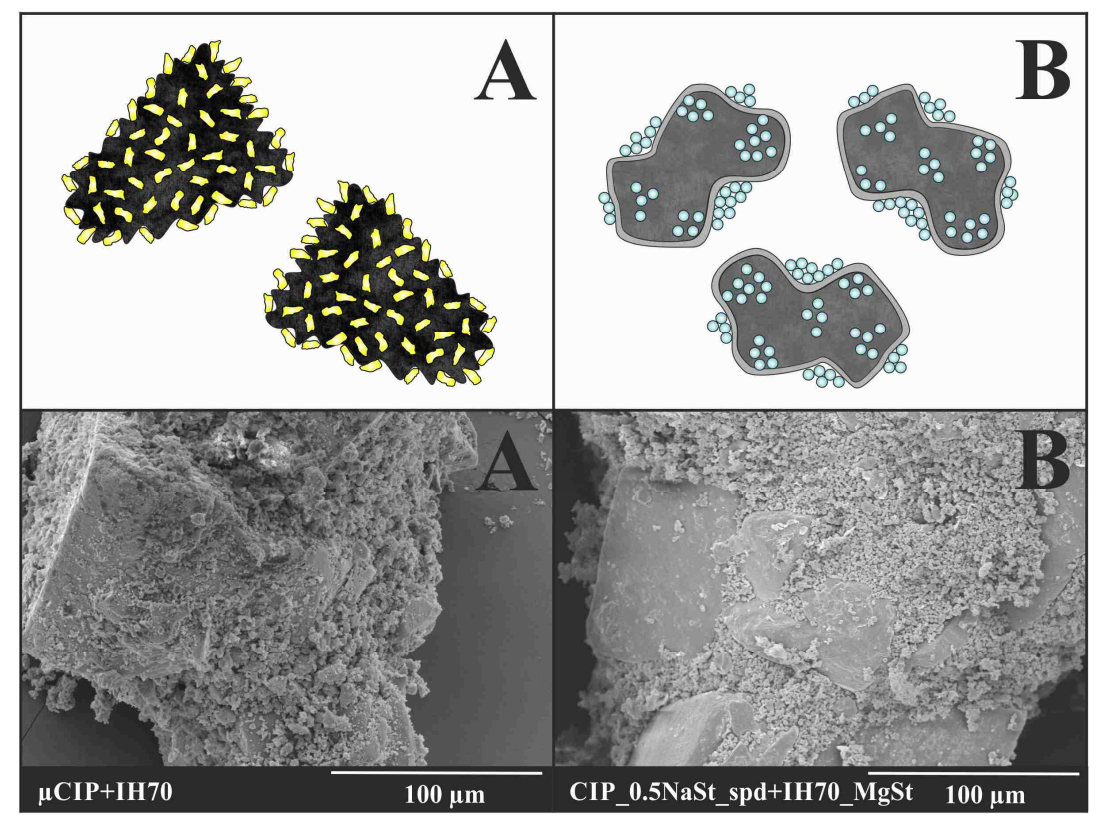

Figure 5. Schematic interpretation of $\mu$ CIP and IH70 after blending (A) and the effect of MgSt-modification on the distribution of co-spray dried CIP (B)

During DPI formulation, two types of interactions should be critical factors, drug-drug cohesive forces (carrier-free formulation) and drug-carrier adhesive forces (carrier-based formulation). First, contact angle measurements were performed to calculate the polarity characteristic of the materials and cohesive work $\left(\mathrm{W}_{\mathrm{c}}\right)$ that can be interpreted between particles of the same quality. The lipophilic components (MgSt and NaSt) affected polarity and cohesivity. The highest polarity values were obtained with crystalline $\mu$ CIP. Concerning the results of cohesion work $\left(\mathrm{W}_{\mathrm{c}}\right)$, it can be stated that in the case of CIP_spd, cohesivity decreased between the spherical, rough particles and the presence of $\mathrm{NaSt}$ could also contribute to the decrease in $\mathrm{W}_{\mathrm{c}}$. This could be the main reason for the topicality of carrier-free systems because the special morphology and the lower cohesivity of particles could result in better deposition properties. As described in the Methods part, we determined the work and force of adhesion and the spreading coefficient (see Table 5.) for the blended products. It can be stated that MgSt reduced the work and force of adhesion (anti-adhesive excipient) and decreased the spreadability of the drug on the surface (a high $S$ value means less spreadability). It was found that the adhesion force in the novel formulated products containing the spray dried drug and the surface modified IH70 was considerably lower than in about one-third of the carrier-based samples containing $\mu \mathrm{CIP}$.

Table 5. Evaluation of adhesive-cohesive balance

\begin{tabular}{lcccc}
\hline Samples & $\begin{array}{c}\mathbf{W}_{\mathbf{c}} \\
{[\mathrm{mN} / \mathrm{m}]}\end{array}$ & $\begin{array}{c}\mathbf{W}_{\text {adh }} \\
{[\mathrm{mN} / \mathrm{m}]}\end{array}$ & $\begin{array}{c}\mathbf{F}_{\text {adh }} \\
{[\mathrm{mN}]}\end{array}$ & $\mathbf{S}_{\mathbf{2 1}}$ \\
\hline $\boldsymbol{\mu C I P}$ & 161.60 & - & - & -
\end{tabular}




\begin{tabular}{lcccc}
\hline $\boldsymbol{\mu C I P + I H 7 0}$ & - & 108.26 & $1.690 * 10^{-3}$ & 1.64 \\
CIP_0.5NaSt_spd & 123.26 & - & - & - \\
CIP_0.5NaSt_spd+IH70_MgSt & - & 72.57 & $0.504 * 10^{-3}$ & -19.06 \\
\hline
\end{tabular}

As a summary of interparticle interactions, we conclude that adhesion is strong between $\mu \mathrm{CIP}$ and IH70 by blending, which resulted in an FPF value of only $23 \%$ with low lung deposition. We observed that co-spray-drying with NaSt decreased cohesivity, the FPF of the carrier-free formulation was almost high (54\%). The novel combined technology could offer less cohesivity and adhesivity, therefore aerodynamic behaviour improved. With the use of $\mathrm{MgSt}$, polarity, the work of adhesion and the force of adhesion decreased, thus spreading became less favourable. Therefore the interparticle interactions were modified, the drug may be easier to detach from the surface of the carrier during inhalation with FPF around $63 \%$.

\section{Conclusion}

Thanks to their beneficial properties, dry powder inhalers (DPI) can have an important role in drug formulation as compared with Nebulizers and pressurized metered dose inhalers. The purpose of our research work was the development of a novel antibiotic-containing DPI including the advantages of carrier-based and carrier-free formulations. The samples containing ciprofloxacin hydrochloride (CIP), lipophilic additive (NaSt), surface modifier (MgSt) and inhalable carrier (IH70) were characterized. Based on the comparison of the results we concluded that cohesive-adhesive balance could be modified with the particle engineering of the drug and the presence of the excipients. Therefore we offer a new possibility in DPI formulation to improve the in vitro-in silico aerodynamic properties. The in silico deposition results were in line with the in vitro measurements and yielded increased lung doses for the sample prepared by the combination method. The present work demonstrates that the novel procedure for the formulation of CIP-DPI offers a more effective therapy for cystic fibrosis with deeper deposition of the drug.

\section{Declaration of interest}

The authors report no conflicts of interest in this work.

\section{Acknowledgement}

This project was supported by GINOP-2.3.2-15-2016-00036 program and ÚNKP-17-4-IIISZTE-3 New National Excellence Program of the Ministry of Human Capacities.

\section{References}

Benke, E., Szabó-Révész, P., Ambrus, R., 2017. Characterization and development opportunities of carrier- based dry powder inhaler systems. Acta Pharm. Hung. 87, 5968.

Buttini, F., Cuoghi, E., Miozzi, M., Rossi, A., Sonvico, F., Colombo, P., 2012. Insulin SprayDried Powder and Smoothed Lactose: A New Formulation Strategy for Nasal and Pulmonary Delivery. Rdd2012 835-840. 
Byrn, S. R., Zografi, G., Chen, X., 2017. Mechanical Properties of Pharmaceutical Materials, in: Byrn, S. R., Zografi, G., Chen, X. (Eds.), Solid State Properties of Pharmaceutical Materials. John Wiley \& Sons, Inc., Hoboken, p. 240.

Cheng, Y.S., 2003. Aerosol deposition in the extrathoracic region. Aerosol Sci. Technol. 37, 659-671. https://doi.org/10.1080/02786820300906

Chvatal, A., Farkas, Á., Balásházy, I., Szabó-Révész, P., Ambrus, R., 2017. Aerodynamic properties and in silico deposition of meloxicam potassium incorporated in a carrier-free DPI pulmonary system. Int. J. Pharm. 520, 70-78. https://doi.org/10.1016/j.ijpharm.2017.01.070

Coccini, D., Alberi, M. D., Busca, A., Schiaretti, F., 2012. Use of magnesium stearate in dry powder formulations for inhalation. U.S. Patent No. 20120082727A1. Date of Patent: 2012.04.05.

Colthorpe, P., Voshaar, T., Kieckbusch, T., Cuoghi, E., Jauernig, J., 2013. Delivery characteristics of a low-resistance dry-powder inhaler used to deliver the long-acting muscarinic antagonist glycopyrronium. J. Drug Assess. 2, 11-16. https://doi.org/10.3109/21556660.2013.766197

da Silva Andrade, F. R., 2015. Self-assembled polymeric micelles as powders for pulmonary administration of insulin.

Della Bella, A., Salomi, E., Buttini, F., Bettini, R., 2017. The role of the solid state and physical properties of the carrier in adhesive mixtures for lung delivery. Expert Opin. Drug Deliv. 5247, 1-10. https://doi.org/10.1080/17425247.2017.1371132

Farkas, Á., Jókay, Á., Balásházy, I., Füri, P., Müller, V., Tomisa, G., Horváth, A., 2016. Numerical simulation of emitted particle characteristics and airway deposition distribution of Symbicort ${ }^{\circledR}$ Turbuhaler ${ }^{\circledR}$ dry powder fixed combination aerosol drug. Eur. J. Pharm. Sci. 93, 371-379. https://doi.org/10.1016/j.ejps.2016.08.036

Farkas, Á., Lewis, D., Church, T., Tweedie, A., Mason, F., Haddrell, A.E., Reid, J.P., Horváth, A., Balásházy, I., 2017. Experimental and computational study of the effect of breath-actuated mechanism built in the NEXThaler®dry powder inhaler. Int. J. Pharm. 533, 225-235. https://doi.org/10.1016/j.ijpharm.2017.09.057

Haefeli-Bleuer, B., Weibel, E.R., 1988. Morphometry of the human pulmonary acinus. Anat. Rec. 220, 401-414. https://doi.org/10.1002/ar.1092200410

Hazare, S., Menon, M., 2009. Improvement of inhalation profile of DPI formulations by carrier treatment with magnesium stearate. Indian J. Pharm. Sci. 71, 725.

Healy, A.M., Amaro, M.I., Paluch, K.J., Tajber, L., 2014. Dry powders for oral inhalation free of lactose carrier particles. Adv. Drug Deliv. Rev. 75, 32-52.

https://doi.org/10.1016/j.addr.2014.04.005

Kadota, K., Nishimura, T., Hotta, D., Tozuka, Y., 2015. Preparation of composite particles of hydrophilic or hydrophobic drugs with highly branched cyclic dextrin via spray drying for dry powder inhalers. Powder Technol. 283, 16-23. https://doi.org/10.1016/j.powtec.2015.05.014

Karimi, K., Pallagi, E., Szabó-Révész, P., Csóka, I., Ambrus, R., 2016. Development of a 
microparticle-based dry powder inhalation formulation of ciprofloxacin hydrochloride applying the quality by design approach. Drug Des. Devel. Ther. 10, 3331-3343. https://doi.org/10.2147/DDDT.S116443

Keller, M., Müller-Walz, R., 2003. Dry powder for inhalation. U.S. Patent No. 6645466B1. Date of Patent: 2003.11.11.

Koblinger, L., Hofmann, W., 1990. Monte Carlo modeling of aerosol deposition in human lungs. Part I: Simulation of particle transport in a stochastic lung structure. J. Aerosol Sci. 21, 661-674. https://doi.org/10.1016/0021-8502(90)90121-D

Lee, H.-J., Lee, H.-G., Kwon, Y.-B., Kim, J.-Y., Rhee, Y.-S., Chon, J., Park, E.-S., Kim, D.W., Park, C.-W., 2018. The role of lactose carrier on the powder behavior and aerodynamic performance of bosentan microparticles for dry powder inhalation. Eur. J. Pharm. Sci. \#pagerange\#. https://doi.org/10.1016/j.ejps.2018.03.004

Nandiyanto, A.B.D., Okuyama, K., 2011. Progress in developing spray-drying methods for the production of controlled morphology particles: From the nanometer to submicrometer size ranges. Adv. Powder Technol. 22, 1-19. https://doi.org/10.1016/j.apt.2010.09.011

Palistra, M., 2008. The influence of Magnesium Stearate and carrier surface on the deposition performace of carrier based Dry Powder Inhaler formulations.

Parlati, C., 2008. Respirable microparticles of aminoglycoside antibiotics for pulmonary administration.

Peng, T., Lin, S., Niu, B., Wang, X., Huang, Y., Zhang, X., Li, G., Pan, X., Wu, C., 2016. Influence of physical properties of carrier on the performance of dry powder inhalers. Acta Pharm. Sin. B 6, 308-318. https://doi.org/10.1016/j.apsb.2016.03.011

Pilcer, G., Wauthoz, N., Amighi, K., 2012. Lactose characteristics and the generation of the aerosol. Adv. Drug Deliv. Rev. 64, 233-256. https://doi.org/10.1016/j.addr.2011.05.003

Pomázi, A., Ambrus, R., Szabó-Révész, P., 2014. Stability testing of meloxicam-containing microparticles. Acta Pharm. Hung. 84, 55-62.

Shalash, A.O., Molokhia, A.M., Elsayed, M.M.A., 2015. European Journal of Pharmaceutics and Biopharmaceutics Insights into the roles of carrier microstructure in adhesive / carrier-based dry powder inhalation mixtures : Carrier porosity and fine particle content. Eur. J. Pharm. Biopharm. 96, 291-303. https://doi.org/10.1016/j.ejpb.2015.08.006

Stass, H., Baumann-Noss, S., Delesen, H., Nagelschmitz, J., Willmann, S., Edginton, a., Staab, D., 2008. Pharmacokinetics of ciprofloxacin PulmoSphere® inhalational powder. J. Cyst. Fibros. 7, S26. https://doi.org/10.1016/S1569-1993(08)60100-9

Tüske, Zs., 2005. Influence of the surface free energy on the parameters of pellets.

Velaga, S.P., Djuris, J., Cvijic, S., Rozou, S., Russo, P., Colombo, G., Rossi, A., 2018. Dry powder inhalers: An overview of the in vitro dissolution methodologies and their correlation with the biopharmaceutical aspects of the drug products. Eur. J. Pharm. Sci. 113, 18-28. https://doi.org/10.1016/j.ejps.2017.09.002

Wong, W., Crapper, J., Chan, H.-K., Traini, D., Young, P.M., 2010. Pharmacopeial 
methodologies for determining aerodynamic mass distributions of ultra-high dose inhaler medicines. J. Pharm. Biomed. Anal. 51, 853-7.

https://doi.org/10.1016/j.jpba.2009.10.011

Yang, X.F., Xu, Y., Qu, D.S., Li, H.Y., 2015. The influence of amino acids on aztreonam spray-dried powders for inhalation. Asian J. Pharm. Sci. 10, 541-548.

https://doi.org/10.1016/j.ajps.2015.08.002 